\title{
Topological solutions in the self-dual Chern-Simons theory: existence and approximation
}

\author{
by \\ Joel SPRUCK* \\ Department of Mathematics, The Johns Hopkins University, Baltimore, MD 21218, U.S.A. \\ and \\ Yisong YANG ${ }^{\dagger}$ \\ School of Mathematics, Institute for Advanced Study, Princeton, NJ 08540, U.S.A.
}

\begin{abstract}
In this paper a globally convergent computational scheme is established to approximate a topological multivortex solution in the recently discovered self-dual Chern-Simons theory in $\mathbf{R}^{2}$. Our method which is constructive and numerically efficient finds the most superconducting solution in the sense that its Higgs field has the largest possible magnitude. The method consists of two steps: first one obtains by a convergent monotone iterative algorithm a suitable solution of the bounded domain equations and then one takes the large domain limit and approximates the full plane solutions. It is shown that with a special choice of the initial guess function, the approximation sequence approaches exponentially fast a solution in $\mathbf{R}^{2}$. The convergence rate implies that the truncation errors away from local regions are insignificant.
\end{abstract}

Key words: Monotone iterations, Sobolev embeddings.

RÉsumé. - Nous présentons dans cet article un algorithme qui converge globalement vers une solution qui est un multi-tourbillon topologique dans la théorie auto-duale de Chern-Simons sur $\mathbf{R}^{2}$. Notre méthode,

\footnotetext{
* Research supported in part by NSF grant DMS-88-02858 and DOE grant DE-FG02-86ER250125.

${ }^{\dagger}$ Research supported in part by NSF grant DMS-93-04580. Current address: Department of Mathematics, Polytechnic University, Brooklyn, NY 11201, U.S.A.

Classification A.M.S. : $35,81$.
} 
qui est constructive et numériquement efficace fournit la solution la plus superconductrice, en ce sens que son champ de Higgs a norme maximum. Nous procédons de la manière suivante: nous obtenons d'abord une solution appropriée des équations dans le cas d'un domaine borné en utilisant une itération monotone convergente, puis en passant à la limite du grand domaine, nous approximons la solution dans tout le plan. Nous montrons que pour un choix particulier de la fonction initiale, la suite d'approximations converge exponentiellement vers une solution dans $\mathbf{R}^{2}$. La rapidité de la convergence entraîne que les erreurs dues à la troncation hors des régions locales sont négligeables.

\section{INTRODUCTION}

It is well-known that unlike magnetic monopoles, which admit electrically charged generalizations called dyons, there are no charged finite-energy (static) vortices in the classical Yang-Mills-Higgs (YMH) models, according to the study of Julia and Zee [12]. Since charged vortices are important in problems such as high-temperature superconductivity, proton decay, and quantum cosmology, an effort has been made to modify the YMH models in order to accomodate finite-energy charged vortex solutions. A consensus has now been reached that the correct framework should be the YMH models with a Chern-Simons (CS) term added. It has been argued by Paul and Khare ([14], [15]) and de Vega and Schaposnik [2] that with the addition of a CS term in the modified models (both abelian and nonabelian), there exist well-behaved finite-energy charged vortices. More recently, the quantum-mechanical meaning of these solutions has also been explored by several authors including Fröhlich and Marchetti ([3], [4]). The main ingredient in the work ([14], [15], [2]) is a reduction of the field equations of motion through the use of a Nielsen-Olesen type [13] radial ansatz to a coupled system of ordinary differential equations. The nonzero CS coupling constant and the structure of the equations imply that a vortex-like solution must carry both magnetic and electric charges. However, a mathematically rigorous existence result for such solutions has not been established due to some difficulties involved in the equations ([14], [2]). Thus the existene of charged vortices in the full YMH-CS models is still an open question.

Recently, the studies of Hong, Kim, and Pac [6] and Jackiw and Weinberg [10] shed new light on the existence problem. It has been argued that in the strong CS coupling limit $(\kappa \rightarrow \infty)$, the influence of the Maxwell term can be neglected from the action and the dynamics is still preserved at 
large distances. The new model admits an interesting reduction similar to that of Bogomol'nyi [1] for the Nielsen-Olesen vortices when the coupling parameters verify a critical condition and the Higgs potential energy density function takes a special form. The equations of motion now become a Bogomol'nyi-type system of the first-order equations which allows the existence of topological solutions. See Wang [18] for a variational proof of existence.

One of the problematical mathematical issues in the vortex models (both classical and CS) is that, unlike monopoles and instantons, there are no known explicit nontrivial solutions. Therefore we have undertaken in this paper to provide a convergent approximation scheme to compute such vortex-type solutions. We will present a monotone iterative method for the computation of the self-dual CS vortices in $\mathbf{R}^{2}$.

The main feature in our approach is that, when the initial function is suitably chosen, the iterative sequence approximates exponentially fast an exact multivortex solution of the CS-Bogomol'nyi system. More precisely, we shall study the scalar semilinear elliptic equation (with source term characterizing the location of the vortices) which is equivalent to the CSBogomol'nyi system. In the first stage of our algorithm, we show that over a bounded domain, a solution may be found as the monotone decreasing limit of an iterative sequence. The sequence has the additional property that it decreases the natural energy functional associated to the scalar semilinear elliptic equation. This property is the key to proving the strong convergence of the sequence and is related to the work of Wang [18]. Next, we show that as the domains are made bigger, the solutions on bounded domains will decrease to a solution in the full $\mathbf{R}^{2}$. In other words, bigger domains provide better approximations. It is interesting to note that the solution obtained this way is "maximal". For example, it gives rise to the largest possible magnitude of the Higgs field (hence we may call such a solution "most superconducting"). This by-product may also give some insight into the uniqueness of the solutions. Since we are approximating a solution in the entire plane, some control of the sequence at infinity must be achieved. For this purpose it is essential to know the asymptotic behavior of finite energy topological solutions in $\mathbf{R}^{2}$. It will be seen that the physical field strengths all approach their limiting values exponentially fast. Such a result is not surprising due to the Higgs mechanism in the model. However it has many important implications including the quantization of flux and charge and has not been discussed in detail in literature. Our convergence result thus obtained is global in $\mathbf{R}^{2}$. It is hoped that the method here might also be applied to the models with larger gauge groups or with 
more fields coupled together, proposed in various latest developments of the subject.

The rest of the paper is organized as follows. In Section 2 we make a short description of the self-dual CS vortex equations and fix most of the notation and state our main result. In Section 3 we present our monotone iterative scheme for computing topological CS vortices and establish the basic properties of the scheme including its uniform convergence. In Section 4 we show that the limit of the finite domain solutions converges strongly to a solution in $\mathbf{R}^{2}$. In particular, we establish that the global solution gives rise to a finite-energy solution of the CS self-dual equations and that the approximating fields converge exponentially fast. In Section 5 we make a short discussion of some numerical solutions of the self-dual CS system.

\section{THE SELF-DUAL TOPOLOGICAL VORTICES}

The Minkowski spacetime metric tensor $g_{\mu \nu}$ is $\operatorname{diag}(1,-1,-1)$. In normalized units and assuming the critical coupling, the Lagrangian density is written

$$
\mathcal{L}=\left(D_{\mu} \phi\right)\left(D^{\mu} \phi\right)^{*}+\frac{1}{4} \kappa \varepsilon^{\alpha \beta \gamma} A_{\alpha} F_{\beta \gamma}-\frac{1}{\kappa^{2}}|\phi|^{2}\left(1-|\phi|^{2}\right)^{2},
$$

where $D_{\mu}=\partial_{\mu}-\mathrm{i} A_{\mu}, \kappa \in \mathbf{R}$ is nonzero, $\varepsilon^{\alpha \beta \gamma}$ is totally skew-symmetric with $\varepsilon^{012}=1, F_{\alpha \beta}=\partial_{\alpha} A_{\beta}-\partial_{\beta} A_{\alpha}$. From the equations of motion of (2.1), we see that the vector $j^{\alpha}=\mathrm{i}\left(\phi\left[D^{\alpha} \phi\right]^{*}-\phi^{*}\left[D^{\alpha} \phi\right]\right)=(\rho, \mathbf{j})$ is the conserved matter current density and $B=F_{12}$ the magnetic field. In the rest of the paper we assume that the field configurations are static. Then the modified Gauss law of the equations of motion of (2.1) reads

$$
\kappa F_{12}=j^{0}=\rho=-2 A_{0}|\phi|^{2} .
$$

As a consequence, there holds the flux-charge relation

$$
\kappa \Phi=\kappa \int_{\mathbf{R}^{2}} F_{12} \mathrm{~d} x=\int_{\mathbf{R}^{2}} \rho \mathrm{d} x=Q .
$$

From calculating the energy-momentum tensor of (2.1) and using the above mentioned Gauss law, we see that the energy density is

$$
\mathcal{E}=\frac{\kappa^{2}}{4} \cdot \frac{F_{12}^{2}}{|\phi|^{2}}+\left|D_{j} \phi\right|^{2}+V(|\phi|) .
$$


It was first found in ([6], [10]) that the equations of motion in the static limit (the CS equations) can be verified by the solutions of (2.2) coupled with the Bogomol'nyi system

$$
\left\{\begin{array}{l}
D_{1} \phi+\mathrm{i} D_{2} \phi=0 \\
F_{12}+\frac{2}{\kappa^{2}}|\phi|^{2}\left(|\phi|^{2}-1\right)=0,
\end{array}\right.
$$

which saturate various quantized energy strata. Solutions of (2.2) and (2.5) are called self-dual CS vortices. If $(\phi, A)$ is a solution so that the energy $\int \mathcal{E}$ is finite where $\mathcal{E}$ is as defined in (2.4), then

$$
\text { either } \phi \rightarrow 0 \quad \text { or } \quad|\phi|^{2} \rightarrow 1 \text { as }|x| \rightarrow \infty \text {. }
$$

The latter is called topological while the former non-topological. This paper concentrates on topological solutions.

The following is our main result.

THEOREM 2.1. - Suppose that $(\phi, A)$ is a finite-energy topological solution of the CS vortex equations (2.2) and (2.5). Then the physical energy terms satisfy the following decay properties at infinity:

$$
\left\{\begin{array}{l}
\left|D_{j} \phi\right|^{2}=O\left(e^{-m_{1}(1-\varepsilon)|x|}\right), \\
1-|\phi|^{2}, \quad F_{12}=O\left(e^{-m_{2}(1-\varepsilon)|x|}\right),
\end{array}\right.
$$

where $m_{1}=2 \sqrt{2} /|\kappa|, m_{2}=2 /|\kappa|$, and $\varepsilon \in(0,1)$ is arbitrary. Besides, there is a non-negative integer $N$ which is the winding number of $\phi$ at a circle near infinity so that the energy $E=\int \mathcal{E}$ and the magnetic flux $\Phi$ and the electric charge $Q$ defined in (2.3) are all quantized:

$$
E=2 \pi N, \quad \Phi=2 \pi N, \quad Q=2 \pi N \kappa .
$$

The integer $N$ is actually the algebraic number of zeros of the Higgs field $\phi$ in $\mathbf{R}^{2}$. Conversely, let $p_{1}, \ldots, p_{m} \in \mathbf{R}^{2}$ and $n_{1}, \ldots, n_{m} \in \mathbf{Z}_{+}$(the set of positive integers). The equations (2.2) and (2.5) have a topological solution $(\phi, A)$ so that the zeros of $\phi$ are exactly $p_{1}, \ldots, p_{m}$ with the corresponding multiplicities $n_{1}, \ldots, n_{m}$ and the conditions in (2.8) are fulfilled with $N=\sum n_{l}$. The solution is maximal in the sense that the Higgs field $\phi$ has the largest possible magnitude among all the solutions realizing the same zero distribution and local vortex charges in the plane. Furthermore, the maximal solution may be approximated by a monotone iterative scheme defined over bounded domains in such a way that the truncation errors away from local regions are exponentially small as a function of the distance. 
The decay property (2.6) follows from the finite-energy condition and the self-dual CS equations (2.5). In fact, (2.5) is an elliptic system. Using an iterated $L^{2}$-estimate argument as in ([11], [17], [21]), we can prove (2.6). By (2.6) and the maximum principle, the exponential decay estimates (2.7) for topological solutions may be established without much effort. Then the quantization condition (2.8) can be directly recognized. All these details are skipped here for brievity. The existence part will be worked out in this paper as a by-product of our approximation scheme. In the paper [18], it appears that the author has found a topological solution. Unfortunately, however, no elaboration on the finiteness of the CS energy or the asymptotic behavior is made there to verify that the solution is indeed topological. Hence we will present our approach in such a way that the construction to follow does not rely on the result in that paper.

\section{THE ITERATIVE COMPUTATIONAL SCHEME}

In this section we present an iterative method for the computation of the CS vortices in $\mathbf{R}^{2}$. We discuss the basic properties of the scheme and then prove its convergence. To obtain an $N$-vortex solution with vortices at $p_{1}, \ldots, p_{m} \in \mathbf{R}^{2}$ and local winding numbers $n_{1}, \ldots, n_{m} \in \mathbf{Z}_{+}$so that $\sum n_{l}=N$, we are to solve the equation

$$
\Delta u=\frac{4}{\kappa^{2}} e^{u}\left(e^{u}-1\right)+4 \pi \sum_{l=1}^{m} n_{l} \delta\left(x-p_{l}\right), \quad x \in \mathbf{R}^{2}
$$

Conversely, if $u$ is a solution of (3.1), then we can construct a solution pair $(\phi, A)$ for (2.2) and (2.5) so that $|\phi|^{2}=e^{u}$ and the zeros of $\phi$ are exactly $p_{1}, \ldots, p_{m}$ with the corresponding multiplicities (or local vortex charges) $n_{1}, \ldots, n_{m}$. Thus we need only to concentrate on (3.1).

Define $u_{0}$ :

$$
u_{0}(z)=-\sum_{l=1}^{m} n_{l} \ln \left(1+\left|x-p_{l}\right|^{-2}\right) .
$$

Then the substitution $v=u-u_{0}$ changes (3.1) into the form

$$
\Delta v=\frac{4}{\kappa^{2}} e^{u_{0}+v}\left(e^{u_{0}+v}-1\right)+g \text { in } \mathbf{R}^{2}
$$


where

$$
g=4 \sum_{l=1}^{m} n_{l}\left(1+\left|x-p_{l}\right|^{2}\right)^{-2}
$$

Our monotone iterative scheme can be described as follows.

Let $\Omega_{0} \subset \mathbf{R}^{2}$ be a fixed bounded domain containing the prescribed zero set $Z(\phi)=\left\{p_{1}, \ldots, p_{m}\right\}$ of the Higgs field $\phi$ and let $\Omega \supset \bar{\Omega}_{0}$ be a bounded domain with sufficiently regular (Lipschitzian, say) boundary. Let $K>0$ be a constant verifying $K \geq 8 / \kappa^{2}$. We first introduce an iteration sequence on $\Omega$ :

$$
\left\{\begin{aligned}
(\Delta-K) v_{n} & =\frac{4}{\kappa^{2}} e^{u_{0}+v_{n-1}}\left(e^{u_{0}+v_{n-1}}-1\right)-K v_{n-1}+g \quad \text { in } \Omega \\
v_{n} & =-u_{0} \quad \text { on } \partial \Omega, \quad n=1,2, \ldots \\
v_{0} & =-u_{0} .
\end{aligned}\right.
$$

LEMMA 3.1. - Let $\left\{v_{n}\right\}$ be the sequence defined by the iteration scheme (3.3). Then

$$
v_{0} \geq v_{1} \geq v_{2} \geq \cdots \geq v_{n} \geq \cdots
$$

Proof. - We prove (3.4) by induction. It is easy to verify that $(\Delta-K)\left(v_{1}-v_{0}\right)=0$ in $\Omega-\left\{p_{1}, \ldots, p_{m}\right\} \underset{m}{\text { and }} v_{1} \in C^{\infty}(\Omega-$ $\left.\left\{p_{1}, \ldots, p_{m}\right\}\right)$. For $\varepsilon>0$ small, set $\Omega_{\varepsilon}=\Omega-\bigcup_{l=1}^{m}\left\{x|| x-p_{l} \mid \leq \varepsilon\right\}$. If $\varepsilon>0$ is sufficiently small, we have $v_{1}-v_{0} \leq 0$ on $\partial \Omega_{\varepsilon}$. Hence the maximum principle implies $v_{1} \leq v_{0}$ in $\Omega_{\varepsilon}$. Therefore $v_{1} \leq v_{0}$ in $\Omega$.

In general, suppose there holds $v_{0} \geq v_{1} \geq \cdots \geq v_{k}$. We obtain from (3.3)

$$
\begin{aligned}
(\Delta-K)\left(v_{k+1}-v_{k}\right)= & \frac{4}{\kappa^{2}} e^{2 u_{0}}\left(e^{2 v_{k}}-e^{2 v_{k-1}}\right) \\
& -K\left(v_{k}-v_{k-1}\right)-\frac{4}{\kappa^{2}} e^{u_{0}}\left(e^{v_{k}}-e^{v_{k-1}}\right) \\
\geq & K\left(e^{2 u_{0}+2 W}-1\right)\left(v_{k}-v_{k-1}\right) \\
& -\frac{4}{\kappa^{2}} e^{u_{0}}\left(e^{v_{k}}-e^{v_{k-1}}\right) \quad\left(v_{k} \leq W \leq v_{k-1} \leq v_{0}\right) \\
\geq & K\left(e^{2 u_{0}+2 u_{0}}-1\right)\left(v_{k}-v_{k-1}\right)=0 .
\end{aligned}
$$

Since $v_{k+1}-v_{k}=0$ on $\partial \Omega$, the maximum principle applied to (3.5) gives $v_{k+1} \leq v_{k}$ in $\Omega$. This proves the lemma. 
Now let

$$
F(v) \equiv \int_{\Omega} \mathrm{d} x\left\{\frac{1}{2}|\nabla v|^{2}+\frac{2}{\kappa^{2}}\left(e^{u_{0}+v}-1\right)^{2}+g v\right\}
$$

be the natural functional associated to the Euler equation (3.2). Then the iterates $\left\{v_{n}\right\}$ enjoy the following monotonicity property.

LEMMA 3.2. - There holds $F\left(v_{n}\right) \leq F\left(v_{n-1}\right) \leq \cdots \leq F\left(v_{1}\right) \leq C$ where $C$ depends only on $\Omega_{0}$.

Proof. - Multiplying (3.3) by $v_{n}-v_{n-1}$ and integrating by parts give

$$
\begin{array}{rl}
\int_{\Omega} \mathrm{d} & x\left\{\left|\nabla v_{n}\right|^{2}-\nabla v_{n} \cdot \nabla v_{n-1}+K\left(v_{n}-v_{n-1}\right)^{2}\right\} \\
= & -\frac{4}{\kappa^{2}} \int_{\Omega} \mathrm{d} x\left\{\left(v_{n}-v_{n-1}\right) e^{u_{0}+v_{n-1}}\left(e^{u_{0}+v_{n-1}}-1\right)\right\} \\
& -\int_{\Omega} g\left(v_{n}-v_{n-1}\right) \mathrm{d} x
\end{array}
$$

Now observe that for $u_{0}+v \leq 0$ and $K \geq 4 / \kappa^{2}$, the function

$$
\varphi(v) \equiv \frac{2}{\kappa^{2}}\left(e^{u_{0}+v}-1\right)^{2}-\frac{K}{2} v^{2}
$$

is concave in $v$. Hence

$$
\text { (3.7) } \begin{aligned}
\frac{2}{\kappa^{2}}\left(e^{u_{0}+v_{n}}-1\right)^{2} \leq & \frac{2}{\kappa^{2}}\left(e^{u_{0}+v_{n-1}}-1\right)^{2}+\frac{K}{2}\left(v_{n}-v_{n-1}\right)^{2} \\
& +\frac{4}{\kappa^{2}}\left(v_{n}-v_{n-1}\right) e^{u_{0}+v_{n-1}}\left(e^{u_{0}+v_{n-1}}-1\right)
\end{aligned}
$$

Using (3.6)-(3.7) and $\left|\nabla v_{n} \cdot \nabla v_{n-1}\right| \leq 1 / 2\left(\left|\nabla v_{n}\right|^{2}+\left|\nabla v_{n-1}\right|^{2}\right)$, we finally obtain

$$
F\left(v_{n}\right)+\frac{K}{2}\left\|v_{n}-v_{n-1}\right\|_{L^{2}(\Omega)}^{2} \leq F\left(v_{n-1}\right)
$$

which is a slightly stronger form of the required monotonicity.

Next we show that $F\left(v_{1}\right)$ can be bounded from above by a constant depending only on $\Omega_{0}$. In fact, since $u_{0}+v_{1}=-v_{0}+v_{1} \leq 0$, we have $\left(e^{u_{0}+v_{1}}-1\right)^{2} \leq\left(u_{0}+v_{1}\right)^{2}$. Therefore

$$
\begin{aligned}
F\left(v_{1}\right) \leq & \frac{1}{2}\left\|\nabla v_{1}\right\|_{L^{2}(\Omega)}^{2}+\frac{4}{\kappa^{2}}\left\|v_{1}\right\|_{L^{2}(\Omega)}^{2} \\
& +\frac{4}{\kappa^{2}}\left\|u_{0}\right\|_{L^{2}\left(\mathbf{R}^{2}\right)}^{2}+\|g\|_{L^{2}\left(\mathbf{R}^{2}\right)}\left\|v_{1}\right\|_{L^{2}(\Omega)},
\end{aligned}
$$


and it suffices to prove that $\left\|v_{1}\right\|_{W^{1,2}\left(\Omega_{1}\right)} \leq C$, where $C>0$ depends only on $\Omega_{0}$. To see this, assume $\tilde{u}_{0} \in C^{\infty}\left(\mathbf{R}^{2}\right)$ be such that $\tilde{u}_{0}=u_{0}$ outside $\Omega_{0}$. Then $\Delta \tilde{u}_{0}=-g+f$ where $f$ is smooth and of compact support. Hence $v_{1}+\tilde{u}_{0}=0$ on $\partial \Omega$ and

$$
(\Delta-K)\left(v_{1}+\tilde{u}_{0}\right)=f+K\left(u_{0}-\tilde{u}_{0}\right) \text { in } \Omega .
$$

Multiplying the above equation by $v_{1}+\tilde{u}_{0}$, integrating by parts, and using the Schwarz inequality, we obtain $\left\|v_{1}\right\|_{W^{1,2}(\Omega)} \leq C$, where $C>0$ depends only on $K,\|f\|_{L^{2}\left(\mathbf{R}^{2}\right)},\left\|u_{0}\right\|_{L^{2}\left(\mathbf{R}^{2}\right)}$, and $\left\|\tilde{u}_{0}\right\|_{W^{1,2}\left(\mathbf{R}^{2}\right)}$.

Using Lemmas 3.2 and a refinement of the argument of Wang [18], we can control the $W^{1,2}\left(\mathbf{R}^{2}\right)$ norm of the sequence.

Proposition 3.3. - There holds $\left\|v_{n}\right\|_{W^{1,2}(\Omega)} \leq C, n=1,2, \ldots$, where $C$ depends only on $\Omega_{0}$.

Proof. - We show that $F(v)$ controls the $W^{1,2}$ norm of $v$. Given $v \in W^{1,2}(\Omega)$ with $v=-u_{0}$ on $\partial \Omega$, define

$$
\tilde{v}= \begin{cases}v & \text { in } \Omega \\ -u_{0} & \text { in } \mathbf{R}^{2}-\Omega\end{cases}
$$

Then $\tilde{v} \in W^{1,2}\left(\mathbf{R}^{2}\right)$ and we have the interpolation inequality

$$
\int_{\mathbf{R}^{2}} \tilde{v}^{4} \mathrm{~d} x \leq 2 \int_{\mathbf{R}^{2}} \tilde{v}^{2} \mathrm{~d} x \int_{\mathbf{R}^{2}}|\nabla \tilde{v}|^{2} \mathrm{~d} x .
$$

This implies

$$
\begin{aligned}
\int_{\Omega} v^{4} \mathrm{~d} x \leq & 2 \int_{\Omega} v^{2} \mathrm{~d} x \int_{\Omega}|\nabla v|^{2} \mathrm{~d} x \\
& +C\left(\int_{\Omega} \mathrm{d} x\left\{|\nabla v|^{2}+v^{2}\right\}\right)+C
\end{aligned}
$$

with uniform constant $C$ approaching zero as $\Omega$ tends to $\mathbf{R}^{2}$.

To estimate $F(v)$ from below we use (3.9) to get

$$
\begin{aligned}
\left|\int_{\Omega} g v \mathrm{~d} x\right| & \leq\|g\|_{L^{4 / 3}(\Omega)}\|v\|_{L^{4}(\Omega)} \leq C\|v\|_{L^{4}(\Omega)} \\
& \leq \varepsilon\|v\|_{L^{2}(\Omega)}+\frac{C}{\varepsilon}\|\nabla v\|_{L^{2}(\Omega)}+C \\
& \leq \varepsilon\|v\|_{L^{2}(\Omega)}+\frac{1}{4} \int_{\Omega}|\nabla v|^{2} \mathrm{~d} x+\frac{C}{\varepsilon^{2}},
\end{aligned}
$$


where (and in the sequel) $C>0$ is a uniform constant which may change its value at different places and $\varepsilon>0$ will be chosen below. Now

$$
\begin{aligned}
\left(e^{u_{0}+v}-1\right)^{2} & \geq \frac{\left|u_{0}+v\right|^{2}}{\left(1+\left|u_{0}+v\right|\right)^{2}} \\
& \geq \frac{\frac{1}{2}|v|^{2}}{\left(1+|v|+\left|u_{0}\right|\right)^{2}}-\frac{\left|u_{0}\right|^{2}}{\left(1+\left|u_{0}\right|\right)^{2}}
\end{aligned}
$$

From (3.10)-(3.11) we obtain the lower bound

$$
\begin{aligned}
F(v) \geq & \frac{1}{4} \int_{\Omega}|\nabla v|^{2} \mathrm{~d} x+\frac{1}{\kappa^{2}} \int_{\Omega} \frac{|v|^{2}}{\left(1+|v|+\left|u_{0}\right|\right)^{2}} \mathrm{~d} x \\
& -\varepsilon\|v\|_{L^{2}(\Omega)}-\frac{C}{\varepsilon^{2}}-C .
\end{aligned}
$$

Again using (3.9) we can estimate

$$
\begin{aligned}
& \left(\int_{\Omega} v^{2} \mathrm{~d} x\right)^{2}=\left(\int_{\Omega} \frac{|v|}{\left(1+|v|+\left|u_{0}\right|\right)}\left(1+|v|+\left|u_{0}\right|\right)|v| \mathrm{d} x\right)^{2} \\
& \leq C \int_{\Omega} \frac{|v|^{2}}{\left(1+|v|+\left|u_{0}\right|\right)^{2}} \mathrm{~d} x \int_{\Omega}\left(|v|^{2}+|v|^{4}+\left|u_{0}\right|^{4}\right) \mathrm{d} x \\
& \leq C \int_{\Omega} \frac{|v|^{2}}{\left(1+|v|+\left|u_{0}\right|\right)^{2}} \mathrm{~d} x \\
& \quad \times\left(\int_{\Omega} v^{2} \mathrm{~d} x+\int_{\Omega} v^{2} \mathrm{~d} x \int_{\Omega}|\nabla v|^{2} \mathrm{~d} x+\int_{\Omega}|\nabla v|^{2} \mathrm{~d} x+1\right) \\
& \leq \frac{1}{2}\left(\int_{\Omega} v^{2} \mathrm{~d} x\right)^{2} \\
& \quad+C\left(\left[\int_{\Omega} \frac{|v|^{2}}{\left(1+|v|+\left|u_{0}\right|\right)^{2}} \mathrm{~d} x\right]^{4}+\left[\int_{\Omega}|\nabla v|^{2} \mathrm{~d} x\right]^{4}+1\right)
\end{aligned}
$$

Hence,

(3.13) $\|v\|_{L^{2}(\Omega)} \leq C\left\{\int_{\Omega} \mathrm{d} x\left(|\nabla v|^{2}+\frac{v^{2}}{\left[1+|v|+\left|u_{0}\right|\right]^{2}}\right)+1\right\}$.

Finally, we obtain from (3.12)-(3.13)

$$
\|v\|_{L^{2}(\Omega)} \leq C\left\{F(v)+\varepsilon\|v\|_{L^{2}(\Omega)}+\frac{1}{\varepsilon^{2}}+1\right\} .
$$


Let $\varepsilon$ be so small that $\varepsilon(C+1)<1$. Thus (3.12) and (3.14) imply the desired bound

$$
\|v\|_{W^{1,2}(\Omega)} \leq C\{F(v)+1\} .
$$

The proposition now follows from (3.15) and Lemma 3.2.

An immediate corollary of Proposition 3.3, Lemma 3.1 and standard elliptic regularity is the uniform convergence of the iteration scheme (3.3) to a smooth solution in any topology. We summarize this basic result as

THEOREM 3.4. - The sequence (3.3) converges to a smooth solution $v$ of the boundary value problem

$$
\left\{\begin{aligned}
\Delta v & =\frac{4}{\kappa^{2}} e^{u_{0}+v}\left(e^{u_{0}+v}-1\right)+g \quad \text { in } \Omega, \\
v & =-u_{0} \text { on } \partial \Omega .
\end{aligned}\right.
$$

The convergence may be taken in the $C^{k+\alpha}(\Omega) \cap W^{1,2}(\Omega)$ topology.

It is worth mentioning that all the results above are valid without change for the limiting case $\Omega=\mathbf{R}^{2}$. To clarify this point, we note that in such a situation the problem (3.16) becomes

$$
\left\{\begin{aligned}
\Delta v & =\frac{4}{\kappa^{2}} e^{u_{0}+v}\left(e^{u_{0}+v}-1\right)+g \text { in } \mathbf{R}^{2}, \\
v & \rightarrow 0 \text { as }|x| \rightarrow \infty .
\end{aligned}\right.
$$

Therefore, (3.3) must formally be replaced by the following iterative scheme in $\mathbf{R}^{2}$ :

$$
\left\{\begin{aligned}
(\Delta-K) v_{n} & =\frac{4}{\kappa^{2}} e^{u_{0}+v_{n-1}}\left(e^{u_{0}+v_{n-1}}-1\right)-K v_{n-1}+g \quad \text { in } \mathbf{R}^{2} \\
v_{n} & \rightarrow 0 \text { as }|x| \rightarrow \infty, \quad n=1,2, \ldots \\
v_{0} & =-u_{0}
\end{aligned}\right.
$$

In analogy to Theorem 3.4 , we have

THEOREM 3.5. - The scheme (3.18) defines a sequence $\left\{v_{n}\right\}$ in $W^{2,2}\left(\mathbf{R}^{2}\right)$ so that (3.4) is fulfilled in $\mathbf{R}^{2}$. As $n \rightarrow \infty, v_{n}$ converges weakly in the space $W^{k, 2}\left(\mathbf{R}^{2}\right)$ for any $k \geq 1$ to a smooth solution of (3.17). In fact this solution is maximal among all possible solutions of (3.17).

Proof. - We proceed by induction. When $n=1,(3.18)$ takes the form

$$
(\Delta-K) v_{1}=K u_{0}+g .
$$

Vol. $12, n^{\circ} 1-1995$. 
Since $u_{0}, g \in L^{2}\left(\mathbf{R}^{2}\right)$ and $\Delta-K: W^{2,2}\left(\mathbf{R}^{2}\right) \rightarrow L^{2}\left(\mathbf{R}^{2}\right)$ is a bijection, therefore (3.19) defines a unique $v_{1} \in W^{2,2}\left(\mathbf{R}^{2}\right)$. Thus we see in particular that $v_{1}$ vanishes at infinity as desired. On the other hand, there holds $(\Delta-K)\left(v_{1}-v_{0}\right)=0$ in the complement of $\left\{p_{1}, \ldots, p_{m}\right\}$. Hence the argument of Lemma 3.1 proves that $v_{0} \geq v_{1}$.

We now assume for some $k \geq 1$ that the scheme (3.18) defines on $\mathbf{R}^{2}$ the functions $v_{1}, \ldots, v_{k}$ so that

$$
v_{1}, \ldots, v_{k} \in W^{2,2}\left(\mathbf{R}^{2}\right) \quad \text { and } \quad v_{0} \geq v_{1} \geq \cdots \geq v_{k} .
$$

We have, in view of (3.20), $u_{0}+v_{k} \leq 0$. Thus $e^{u_{0}+v_{k}} \leq 1$ and

$$
\left|e^{u_{0}+v_{k}}-1\right| \leq\left|u_{0}+v_{k}\right| \text {. }
$$

As a consequence, for $n=k+1$, the right-hand side of the first equation in (3.18) lies in $L^{2}\left(\mathbf{R}^{2}\right)$ and thus the equation determines a unique $v_{k+1} \in W^{2,2}\left(\mathbf{R}^{2}\right)$. From the fact that $v_{k+1}-v_{k}$ verifies (3.5) and vanishes at infinity, we arrive at $v_{k+1} \leq v_{k}$. Therefore (3.20) is true for any $k$.

By virtue of (3.21), the functional $F(v)$ is finite for $v=v_{k}, k=1,2, \ldots$ Thus applying Lemma 3.2 and Proposition 3.3 to the sequence $\left\{v_{n}\right\}$ here yields the bound $\left\|v_{n}\right\|_{W^{1,2}\left(\mathbf{R}^{2}\right)} \leq C, n=1,2, \ldots$, where $C>0$ is a constant. Combining this result with (3.21) and using the $L^{2}$-estimates in (3.18), we get $\left\|v_{n}\right\|_{W^{2,2}\left(\mathbf{R}^{2}\right)} \leq C$. In fact a standard bootstrap argument shows that in general one has $\left\|v_{n}\right\|_{W^{k, 2}\left(\mathbf{R}^{2}\right)} \leq C, n \geq$ some $n(k) \geq 1$, where $C>0$ is a constant depending only on $k \geq 1$. Therefore we see that there is a function $v$ so that $v_{n}$ converges weakly in $W^{k, 2}\left(\mathbf{R}^{2}\right)$ for any $k \geq 1$ to $v$ and $v$ is a solution of (3.17).

Finally we show that $v$ is maximal. Let $w$ be another solution of (3.17). Since $-v_{0}+w=0$ at infinity,

(3.22) $\Delta\left(-v_{0}+w\right)=\frac{4}{\kappa^{2}} e^{-u_{0}+w}\left(e^{-v_{0}+w}-1\right)$ in $\mathbf{R}^{2}-\left\{p_{1}, \ldots, p_{m}\right\}$,

and $-v_{0}+w<0$ in a small neighborhood of $\left\{p_{1}, \ldots, p_{m}\right\}$, applying the maximum principle in (3.22) leads to $v_{0} \geq w$. From this fact we can use induction as in the proof of Lemma 4.1 in the next section to establish the general inequality $v_{n} \geq w, n=0,1,2, \ldots$ Hence $v=\lim v_{n} \geq w$ and the theorem follows.

The above theorem says that a solution of (3.2) on the full plane may be constructed via our iterative scheme (3.18). However, from the point of view of computation it is preferable to give a global convergence result so that a full plane solution can be approximated by the solutions of the system restricted to bounded domains. This will be accomplished in the next section. 


\section{GLOBAL CONVERGENCE RESULTS}

In this section we assume the notation used in Section 3. In particular, $\Omega$ denotes a bounded domain.

LEMMA 4.1. - Let $V \in C^{2}(\Omega) \cap C^{0}(\bar{\Omega})$ be such that

$$
\left\{\begin{aligned}
\Delta V & \geq \frac{4}{\kappa^{2}} e^{u_{0}+V}\left(e^{u_{0}+V}-1\right)+g \text { in } \Omega \\
V & \leq-u_{0} \quad \text { on } \partial \Omega
\end{aligned}\right.
$$

and $\left\{v_{n}\right\}$ be the sequence defined in (3.3). Then

$$
v_{0} \geq v_{1} \geq v_{2} \geq \cdots \geq v_{n} \geq \cdots \geq V
$$

Proof. - We prove (4.2) by induction. Note that $v_{0} \geq V$ in $\bar{\Omega}$. For such an inequality already holds on $\partial \Omega$ by the definition of $v_{0}$ and for small $\varepsilon>0, u_{0}+V \leq 0$ on $\partial \Omega_{\varepsilon}$. Hence, the result follows from the maximum principle applied to the inequality

$$
\left\{\begin{aligned}
\Delta\left(u_{0}+V\right) & \geq \frac{4}{\kappa^{2}} e^{u_{0}+V}\left(e^{u_{0}+V}-1\right) \text { in } \Omega_{\varepsilon} \\
u_{0}+V & \leq 0 \text { on } \partial \Omega_{\varepsilon} .
\end{aligned}\right.
$$

Suppose there holds $v_{k} \geq V(k=0,1,2, \ldots)$. We need to show that $v_{k+1} \geq V$. In fact, from (3.3) and (4.1), we get

$$
\begin{aligned}
(\Delta-K)\left(v_{k+1}-V\right) \leq & \frac{4}{\kappa^{2}} e^{2 u_{0}}\left(e^{2 v_{k}}-e^{2 V}\right)-K\left(v_{k}-V\right) \\
& -\frac{4}{\kappa^{2}} e^{u_{0}}\left(e^{v_{k}}-e^{V}\right) \\
\leq & K\left(e^{2 u_{0}+2 W}-1\right)\left(v_{k}-V\right) \quad\left(V \leq W \leq v_{k}\right) \\
\leq & K\left(e^{2 u_{0}+2 v_{0}}-1\right)\left(v_{k}-V\right)=0 .
\end{aligned}
$$

Since for $k+1=n=1,2, \ldots$, the right-hand side of (3.3) always lies in $L^{p}(\Omega)$ for any $p \geq 2$, we see that $v_{k+1} \in W^{2, p}(\Omega)$. In particular $v_{k+1} \in C^{1+\alpha}(\bar{\Omega})(\alpha: 0<\alpha<1)$. On the other hand, we have $v_{k+1}-V \geq 0$ on $\partial \Omega$. Thus (4.3) and the weak maximum principle (see Gilbarg and Trudinger [5]) imply that $v_{k+1} \geq V$ in $\Omega$. The lemma is proved. 
Next, let $\left\{\Omega_{n}\right\}$ be a monotone sequence of bounded convex domains in $\mathbf{R}^{2}$ satisfying the same properties as those for $\Omega$ in defining the iterative scheme (3.3): $\Omega_{1} \subset \Omega_{2} \subset \cdots \subset \Omega_{n} \subset \cdots, \bigcup_{n=1}^{\infty} \Omega_{n}=\mathbf{R}^{2}$.

Lemma 4.2. - Let $v^{(j)}$ and $v^{(k)}$ be the solutions of (3.16) obtained from (3.3) by setting $\Omega=\Omega_{j}$ and $\Omega=\Omega_{k}$ respectively, $j, k=1,2, \ldots$ If $\Omega_{j} \subset \Omega_{k}$, then

$$
v^{(j)} \geq v^{(k)} \quad \text { in } \Omega_{j}
$$

Proof. - By the construction of $v^{(k)}$, we have in particular that $v^{(k)} \leq-u_{0}$ in $\Omega_{k}$. Thus $v^{(k)}$ is a subsolution of (3.16) for $\Omega=\Omega_{j}$. Thus by Lemma 4.1 , we get $v^{(j)} \geq v^{(k)}$ in $\Omega_{j}$.

For convenience, from now on we extend the domain of definition of each $v^{(j)}$ to the entire $\mathbf{R}^{2}$ by setting $v^{(j)}=-u_{0}$ in $\mathbf{R}^{2}-\Omega_{j}$. Thus $\left\{v^{(j)}\right\}$ is a sequence in $W^{1,2}\left(\mathbf{R}^{2}\right)$.

From Proposition 3.3, we can obtain a constant $C>0$ independent of $j=1,2, \ldots$, so that $\left\|v^{(j)}\right\|_{W^{1,2}\left(\mathbf{R}^{2}\right)} \leq C$. As in Section 3, this leads to

THEOREM 4.3. - The sequence of solutions $\left\{v^{(j)}\right\}$ defined in Lemma 4.2 converges weakly in $W^{1,2}\left(\mathbf{R}^{2}\right)$ to the maximal solution of (3.17) obtained in Theorem 3.5 .

Proof. - Let $w$ be the weak limit of the sequence $\left\{v^{(j)}\right\}$ in $W^{1,2}\left(\mathbf{R}^{2}\right)$. Then $w$ is a solution of (3.2) satisfying $u_{0}+w \leq 0$. Hence, as in the proof of Theorem 3.5, the right-hand side of (3.2) now lies in $L^{2}\left(\mathbf{R}^{2}\right)$. But $w \in W^{1,2}\left(\mathbf{R}^{2}\right)$, so the $L^{2}$-estimates applied in (3.2) give the result $w \in W^{2,2}\left(\mathbf{R}^{2}\right)$. Thus we see that $w=0$ at infinity. In particular $w$ is a solution of the problem (3.17). On the other hand, let the maximal solution of (3.17) be $v$. Then $v \geq w$. Recall that the proof of Theorem 3.5 has given us the comparison $u_{0}+v=-v_{0}+v \leq 0$ in $\mathbf{R}^{2}$. So $v$ verifies (4.1) on each $\Omega=\Omega_{j}$. As a consequence, Lemma 4.1 implies that $v^{(j)} \geq v$ in $\Omega_{j}, j=1,2, \ldots$ Therefore $w=\lim v^{(j)} \geq v$. This proves the desired result $v=w$.

In the sequel we shall denote by $\bar{v}$ the maximal solution of (3.17) obtained in Theorem 3.5 or 4.3 and set $\bar{u}=u_{0}+\bar{v}$. Therefore we can construct a finite energy solution pair $(\bar{\phi}, \bar{A})$ of $(2.2)$ and (2.5) so that $|\bar{\phi}|^{2}=e^{\bar{u}}$. In fact we can state

PROPOSITION 4.4. - Let $u=u_{0}+v$ where $v$ is a solution of (3.2) which lies in $W^{2,2}\left(\mathbf{R}^{2}\right)$. Denote by $(\phi, A)$ the solution pair of (2.2) and (2.5) 
constructed by the scheme in [11] so that $|\phi|^{2}=e^{u}$. Then $(\phi, A)$ is of finite energy.

Proof. - By $v \in W^{2,2}\left(\mathbf{R}^{2}\right)$, we see that $v \rightarrow 0$ at infinity. In particular, $\lim _{|x| \rightarrow \infty} u=0$. Thus using the fact that $u<0$ in a neighborhood of $\left\{p_{1}, \ldots, p_{m}\right\}$ and the maximum principle in (3.1) we have $u \leq 0$ in $\mathbf{R}^{2}$. This implies $|\phi|^{2}=e^{u} \leq 1$.

Given $0<\varepsilon<1$, choose $t>0$ sufficiently large to make

$$
\frac{4}{\kappa^{2}} e^{2 u} \geq \frac{4}{\kappa^{2}}(1-\varepsilon)^{2}, \quad|x| \geq t
$$

Set $m_{2}=2 /|\kappa|$. Then from (3.1) we arrive at

$$
\left\{\begin{array}{rrr}
\Delta u & =m_{2}^{2} e^{u} e^{w} u & (u \leq w \leq 0) \\
& \leq m_{2}^{2}(1-\varepsilon)^{2} u, & |x| \geq t
\end{array}\right.
$$

From (4.5) we can show by the maximum principle that there is $C(\varepsilon)>0$ so that

$$
0 \geq u \geq-C(\varepsilon) e^{-m_{2}(1-\varepsilon)|x|}
$$

Hence $|\phi|^{2}-1=e^{u}-1 \in L\left(\mathbf{R}^{2}\right)$.

Since $(\phi, A)$ is a solution of $(2.5)$, $\phi$ verifies the equation

$$
D_{j} D_{j} \phi=\frac{2}{\kappa^{2}}|\phi|^{2}\left(|\phi|^{2}-1\right) \phi \text {. }
$$

For any $\psi \in W_{0}^{1,2}(\Omega)$ where $\Omega \subset \mathbf{R}^{2}$ is a bounded domain, we get by multiplying both sides of the above by $\psi^{*}$ and integrating the equation

$$
\operatorname{Re} \int_{\mathbf{R}^{2}} \mathrm{~d} x\left\{\left(D_{j} \phi\right)\left(D_{j} \psi\right)^{*}+\frac{2}{\kappa^{2}}|\phi|^{2}\left(|\phi|^{2}-1\right) \phi \psi^{*}\right\}=0
$$

Therefore, replacing $\psi$ above by $\eta_{t}^{2} \phi$, we arrive at

$$
(4.6)\left\{\begin{aligned}
0= & \operatorname{Re} \int_{\Omega_{2 t}} \mathrm{~d} x\left\{\left(D_{j} \phi\right)\left(D_{j}\left[\eta_{t}^{2} \phi\right]\right)^{*}+\frac{2}{\kappa^{2}}|\phi|^{4}\left(|\phi|^{2}-1\right) \eta_{t}^{2}\right\} \\
= & 2 \operatorname{Re} \int_{t \leq|x| \leq 2 t} \mathrm{~d} x\left\{\left(D_{j} \phi\right) \eta_{t} \phi^{*} \eta^{\prime}\left(\frac{|x|}{t}\right) \frac{x_{j}}{t|x|}\right\} \\
& +\int_{\Omega_{2 t}} \mathrm{~d} x\left\{\eta_{t}^{2}\left|D_{j} \phi\right|^{2}+\frac{2}{\kappa^{2}}|\phi|^{4}\left(|\phi|^{2}-1\right) \eta_{t}^{2}\right\} .
\end{aligned}\right.
$$


Here $\eta_{t}$ is defined by

$$
\eta_{t}(x)=\eta\left(\frac{|x|}{t}\right), \quad x \in \mathbf{R}^{2}, \quad t>0,
$$

where $\eta \in C_{0}^{\infty}(\mathbf{R})$ is such that

$$
0 \leq \eta \leq 1, \quad \eta(s)=1 \quad \text { for } s \leq 1, \quad \eta(s)=0 \text { for } s \geq 2
$$

Using $|\phi| \leq 1$ and a simple interpolation inequality, (4.6) leads to

$$
\int_{\Omega_{2 t}} \eta_{t}^{2}\left|D_{j} \phi\right|^{2} \mathrm{~d} x \leq C_{1}+\left.C_{2} \int_{\mathbf{R}^{2}}|| \phi\right|^{2}-1 \mid \mathrm{d} x
$$

where $C_{1}, C_{2}>0$ are independent of $t>0$. Letting $t \rightarrow \infty$ in (4.7) we see that $D_{j} \phi \in L^{2}\left(\mathbf{R}^{2}\right)$.

Moreover, from (2.2) and (2.5), we have

$$
A_{0}^{2}|\phi|^{2}, \quad V(|\phi|) \leq \frac{1}{\kappa^{2}}\left(e^{u}-1\right)^{2} \in L\left(\mathbf{R}^{2}\right) .
$$

Consequently $(\phi, A)$ is indeed of finite energy [see (2.4)].

Now let $\left(\phi^{(j)}, A^{(j)}\right)$ be the solution pair of the truncated Bogomol'nyi equations

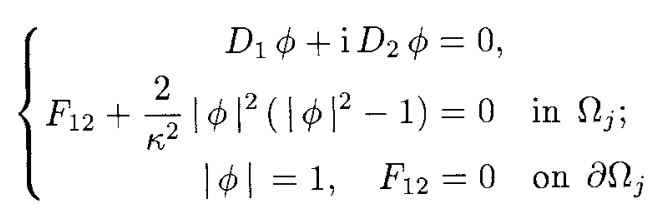

obtained from the function $v^{(j)}$ described in Lemma 4.2. For convenience, we understand that $\left|\phi^{(j)}\right|=1, F_{12}^{(j)}=0$ in $\mathbf{R}^{2}-\Omega_{j}$. Such an assumption corresponds to the earlier extension of $v^{(j)}$ with setting $v^{(j)}=-u_{0}$ in $\mathbf{R}^{2}-\Omega_{j}$. In the sequel, this convention is always implied unless otherwise stated.

Define the norm ||$_{\mu}$ where $\mu \in(0,2 /|\kappa|)$ by

$$
|\eta|_{\mu}=\sup _{x \in \mathbf{R}^{2}}\left|e^{\mu|x|} \eta(x)\right| \text {. }
$$

This expression says functions with finite ||$_{\mu}$ norms decay exponentially fast at infinity. Our global convergence thereom for the computation of a topological solution of (2.2) and (2.5) may be stated as follows. 
THEOREM 4.5. - Let $(\phi, A)$ be an artibrary topological solution of the CS-Bogomol'nyi equations (2.2) and (2.5) with $Z(\phi)=\left\{p_{1}, \ldots, p_{m}\right\}$ and the multiplicities of the zeros $p_{1}, \ldots, p_{m}$ are $n_{1}, \ldots, n_{m} \in \mathbf{Z}_{+}$, respectively, and $\left\{\left(\phi^{(j)}, A^{(j)}\right)\right\}$ be the solution sequence of (4.8) described above. Then $(\bar{\phi}, \bar{A})=\lim _{j \rightarrow \infty}\left(\phi^{(j)}, A^{(j)}\right)$ is a topological solution of $(2.2)$ and (2.5) characterized by the same vortex distribution as $(\phi, A)$ and verifying $|\bar{\phi}| \geq|\phi|$ in $\mathbf{R}^{2}$. Furthermore, the physical fields have the following convergence rate for any $\mu \in(0,2 /|\kappa|)$ :

$$
\left.|| \phi^{(j)}\right|^{2}-\left.|\bar{\phi}|^{2}\right|_{\mu} \rightarrow 0, \quad\left|F_{12}^{(j)}-\bar{F}_{12}\right|_{\mu} \rightarrow 0 \quad \text { as } j \rightarrow \infty .
$$

In particular,

$$
\lim _{j \rightarrow \infty} \int_{\Omega_{j}} F_{12}^{(j)} \mathrm{d} x=\int_{\mathrm{R}^{2}} \bar{F}_{12} \mathrm{~d} x=2 \pi N
$$

where $N=n_{1}+\cdots+n_{m}$.

Proof. - We have already seen in Theorem 4.3 and Proposition 4.4 that the $\bar{v}=\lim _{j \rightarrow \infty} v^{(j)}$ is the maximal solution of (3.17) which generates a finite energy solution pair $(\bar{\phi}, \bar{A})=\lim _{j \rightarrow \infty}\left(\phi_{j}, A_{j}\right)$ of (2.2) and (2.5). We observe that if $(\phi, A)$ is any finite energy topological solution of (2.2) and (2.5), then $|\phi|=1$ at infinity. Thus $v=\ln |\phi|^{2}-u_{0}$ verifies (3.17). Therefore $\bar{v} \geq v$ in $\mathbf{R}^{2}$. Consequently $|\bar{\phi}| \geq|\phi|$.

For $\mu \in(0,2 /|\kappa|)$, choose $\varepsilon \in(0,1)$ to make $(2 /|\kappa|)(1-\varepsilon)>\mu$. Then the fact $\left.|| \phi^{(j)}\right|^{2}-\left.|\bar{\phi}|^{2}\right|_{\mu} \rightarrow 0$ as $j \rightarrow \infty$ follows immediately from the decay estimates (2.7) since

$$
\left|e^{u(j)}-e^{\bar{u}}\right| \leq 1-e^{\bar{u}}=1-|\bar{\phi}|^{2} .
$$

By virtue of the second equation in (2.5), it is straightforward that $\left|F_{12}^{(j)}-\bar{F}_{12}\right| \rightarrow 0$ as $j \rightarrow \infty$.

The proof of Theorem 4.5 is complete.

Note. - An analogue of the above convergence theorem for computing the classical self-dual abelian YMH vortices where the governing equations assume a simpler form has been obtained earlier in Wang and Yang [19]. 


\section{NUMERICAL EXAMPLES}

To test the efficiency of our iterative scheme for computing a topological vortex-like solution of the CS-Bogomol'nyi equations (2.2) and (2.5), here we present several numerical examples. For definiteness, we shall fix the CS coupling parameter $\kappa: \kappa=2$. A multivortex solution of (2.5) on $\mathbf{R}^{2}$ will be obtained by using a solution sequence over bounded domains as illustrated in Section 3. We shall take $\Omega$ to be a square domain: $\Omega=(-a, a) \times(-a, a)$ and discretize it by equidistant grid points, which results in a finite-difference mesh. We then implement the standard fivepoint approximation algorithm for the boundary value problems of elliptic differential equations to obtain a numerical solution of (3.3) at each iteration step $k=n$. As usual, the discrete approximation to $v_{n}$ in (3.3), and so on, at the mesh point $\left(x_{1}(i), x_{2}(j)\right)$, will be denoted by $v_{n}^{i j}$. We choose $K$ in (3.3) to be $K=4$ and compute a multivortex solution with vortices concentrated at $p_{1}=-(\sqrt{15}, \sqrt{15})$ and $p_{2}=(\sqrt{15}, \sqrt{15})$ with unit local charges $n_{1}=n_{2}=1$. This is a two-vortex solution so that the flux verifies $\Phi / 2 \pi=2$. Since $\sqrt{15}<4$, we may choose $a>5$ to ensure that $p_{1}, p_{2} \in \Omega$. When $a$ is small, there is no need to discretize the domain with a large number of mesh points and the computations can be completed rather quickly. However, when $a$ is large, we have to discretize the domain with sufficiently many mesh points to achieve a small discretization error which will result in a longer computing time. To keep a suitable balance, in our range of numerical examples in this section where $a$ has the restriction $5<a<16$, we use 450 points to discretize the interval $(-a, a)$. Experiments show that such a choice already yields satisfactory results. The vortex locations $p_{1}$ and $p_{2}$ are singular points of the scheme (3.3) at the initial step. Due to the smoothing effect in the continuous equations, no problem arises in our theory. In the numerical implementation, since $p_{1}$ and $p_{2}$ are irrational which cannot be mesh points, so no problem arises in the discretized version either. Throughout our computations the stopping criterion of the iterative algorithm (3.3) is set to be

$$
\left|v_{n}-v_{n-1}\right| \equiv \max _{i, j}\left|v_{n}^{i, j}-v_{n-1}^{i, j}\right|<0.002
$$

If the accuracy (5.1) is fulfilled at a certain step $n=k$, then the computation will terminate and $v_{k}^{i, j}$ will be accepted as an approximation of the solution of (3.16) stated in Theorem 3.4 at the mesh points. By virtue of the scheme given in [11], an approximation to a multivortex solution of the truncated CS-Bogomol'nyi equations (4.8) is obtained (with $\Omega=\Omega_{j}$ ). Finally, from 
the discussion in Section 4, a solution of (2.2) and (2.5) over the full plane may be found in the large $\Omega$-domain limit. The examples in the sequel confirm very well our analysis. For simplicity, we shall only present the computer solutions of the magnetic field:

$$
F_{12}=\frac{1}{2}|\phi|^{2}\left(1-|\phi|^{2}\right), \quad|\phi|^{2}=e^{u_{0}+v}
$$

where $v$ is a solution of (3.2) or (3.16). There holds $0 \leq|\phi| \leq 1$. Therefore $0 \leq F_{12} \leq H_{0}$ with $H_{0}=0.125$ [see (5.2)]. The magnetic field of all of our computed solutions attains such a maximum value $\left(H_{0}\right)$ in the domain. Throughout this section, $\pi$ is replaced by its approximation $\pi \approx 3.1415926$.

EXAMPLE 5.1. - We first compute the solution in $\Omega=(-a, a) \times(-a, a)$ with $a=6$. The scheme stops after 36 iterations $(k=36)$ and yields a solution with the flux $\Phi_{\Omega}=0.928452$, where

$$
\Phi_{\Omega}=\frac{1}{2 \pi} \int_{\Omega} F_{12} \mathrm{~d} x,
$$

which is far away from the quantized value $\Phi / 2 \pi=2.0$ in $\mathbf{R}^{2}$. Thus $a=6$ cannot yet provide a good approximation to an $\mathbf{R}^{2}$ solution.

Figure 1 presents a solution with $a=8$. The iteration terminates after 56 steps and turns out a very reasonable flux value: $\Phi_{\Omega}=1.65714$. It may be expected that, as one increases $a$, the solution should stabilize in the local regions around the vortex locations $p_{1}$ and $p_{2}$ and an even better approximation to the flux might be achieved.

EXAMPLE 5.2. - We now take $a=10$. The computation stops at $k=59$. The space distribution of the magnetic field is as shown in Figure 2. It is seen that the solution is indeed stabilized around the vortex concentrations and in the regions some distance away from the centers of vortices the field becomes quite flat. Such a result describes an early stage of the convergence process (as one enlarges the domain) proved in Theorem 4.5. The flux is now greatly improved to $\Phi_{\Omega}=1.91499$.

EXAMPLE 5.3. - We have also found solutions for $a$ in the range $10<a<16$. The computations all stop after the same number of iterations: $k=59$. However, larger $a$ always yields a better flux value. In particular, for $a \leq 12$, the solutions are nicely localized in the neighborhoods of vortices and become almost flat in the regions away from these neighborhoods. Different $a$ 's can no longer give significantly different local behavior of the solutions and the field only assumes zero numerical value in those regions far away from the centers of vortices. Therefore we have observed a steady 


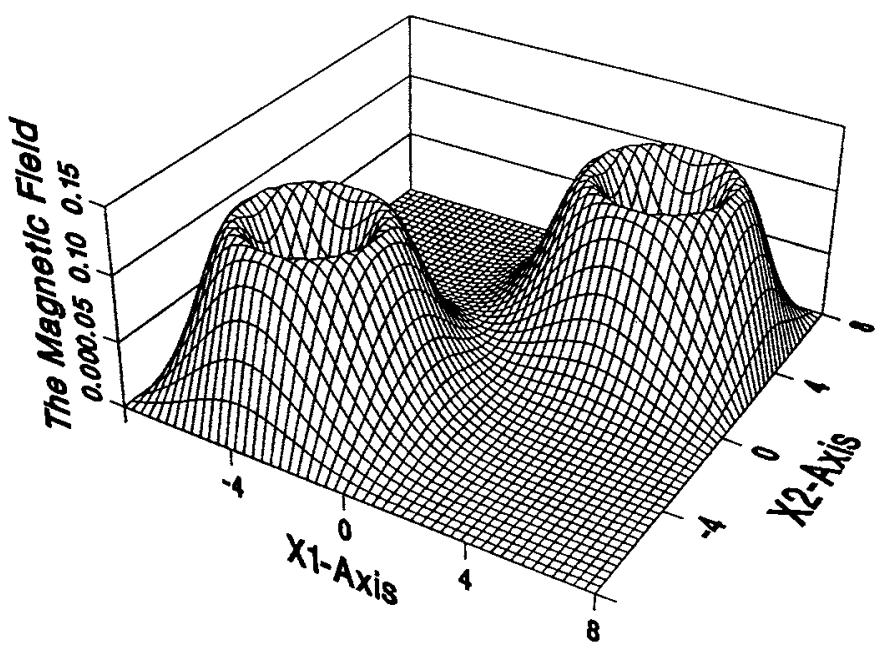

FIG. 1. - A two-vortex solution of the truncated CS-Bogomol'nyi equations. Vortices are concentrated at $\left(x_{1}, x_{2}\right)= \pm(\sqrt{15}, \sqrt{15})$ and carry unit local charges. The solution is obtained after 56 iterations. The flux takes the values $\Phi / 2 \pi=1.65714$.

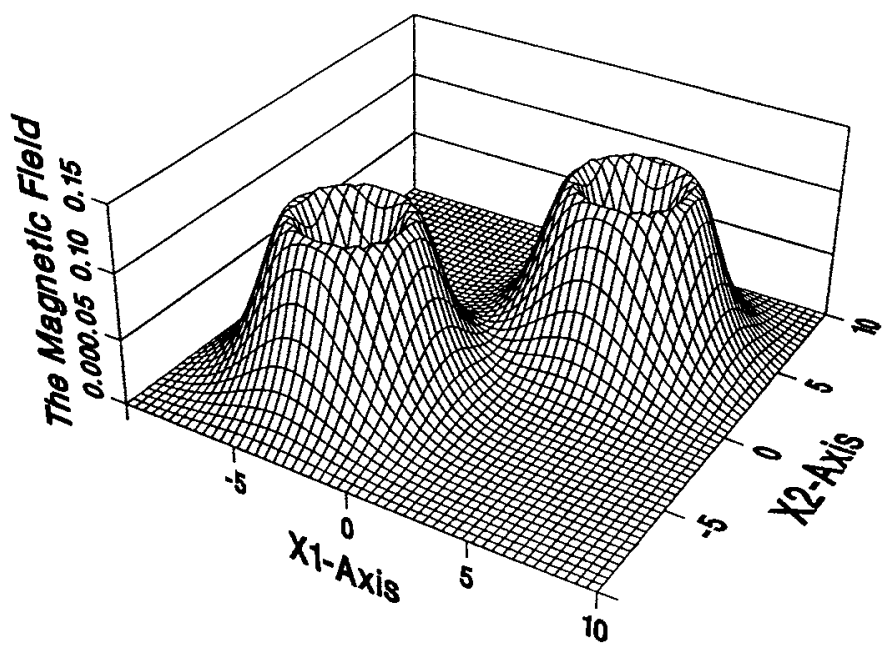

FiG. 2. - An early stage of the convergence of bounded domain solutions to a topological solution in $\mathbb{B}^{2}$. The solution is obtained from the scheme (6.7) after 59 iterations. Vortices have the same local properties as those in Figure 1. In the regions some distance away from the centers of vortices, the field becomes insignificantly flat. The flux is $\Phi / 2 \pi=1.91499$.

convergence process of bounded domain solutions to a solution in $\mathbf{R}^{2}$ and the conclusions in Theorem 4.5 is confirmed.

Figure 3 shows a solution with $a=14$. The flux is $\Phi_{\Omega}=1.96307$. The behavior of the field and the flux value suggest that the $a=14$ solution 
might be accepted as an approximation to a full plane solution of the CS-Bogomol'nyi system (2.2) and (2.5).

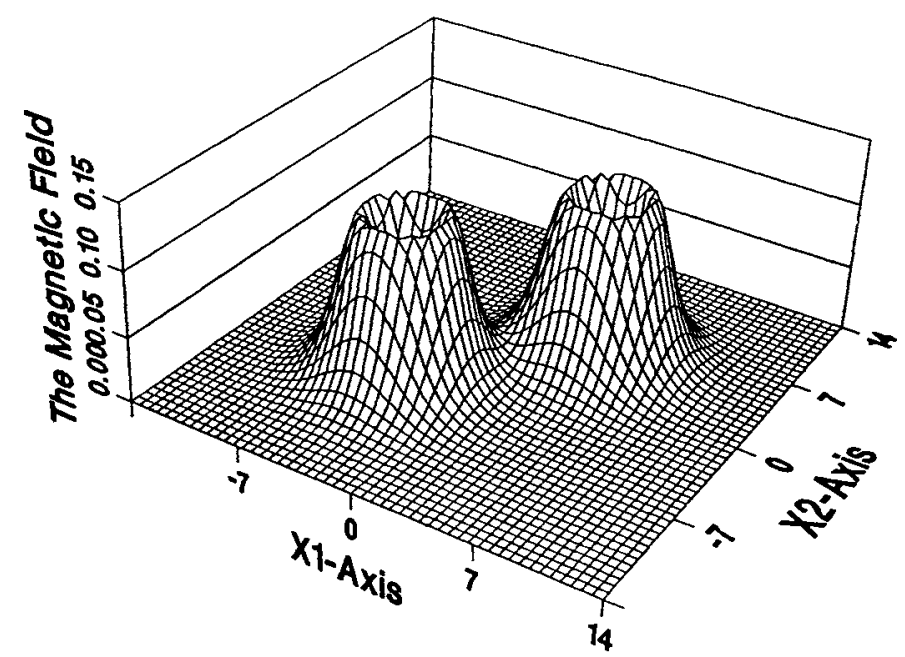

FIG. 3. - Convergence and localization of the two-vortex solution in the large domain limit. This can be viewed as a numerical approximation of a topological solution in $\mathbb{R}^{2}$ with unit vortices at $\left(x_{1}, x_{2}\right)= \pm(\sqrt{15}, \sqrt{15})$. The flux through the square region is $\Phi / 2 \pi=1.96307$. The computation requires 59 iterations.

For $a=15$, the flux takes the value $\Phi_{\Omega}=1.96376$, which slightly improves that with $a=14$.

Figure 3 also suggests that, when the vortices are far apart, a multivortex solution could roughly be viewed as superimposed single-vortex (symmetric) solutions.

The above examples illustrate exactly the established global convergence results of the paper and thus show that our algorithm can be used as a reliable and efficient computational tool in practice to obtain a topological multivortex solution of the self-dual CS theory. According to Theorem 4.5, the solutions found this way are "most superconducting" in the sense that they give rise to the maximal densities of the Cooper pairs when $\phi$ is interpreted as an order parameter in the context of superconductivity theory.

The solutions exhibits themselves quite differently from those in the classical abelian Higgs theory. One of the major distinctions is that in the CS case the magnetic field cannot make any penetration through either the normal or completely superconducting regions characterized by $|\phi|^{2}=0$ or $|\phi|^{2}=1$ and the maximal magnetic excitations occur in the regions in which $|\phi|^{2}=1 / 2$. In fact, this phenomenon is already implied by 
the second equation in (2.5) or (5.2) due to the special form of the potential energy density of the Higgs field, in which both the symmetric and asymmetric vacua are present.

Note. - The solutions of the anti-self-dual CS system

$$
\left\{\begin{array}{l}
D_{1} \phi-\mathrm{i} D_{2} \phi=0 \\
F_{12}-\frac{2}{\kappa^{2}}|\phi|^{2}\left(|\phi|^{2}-1\right)=0
\end{array}\right.
$$

can be obtained from the solutions of the self-dual equations (2.5) by taking "conjugate" $(\phi, A) \mapsto\left(\phi^{*},-A\right)$.

\section{ACKNOWLEDGEMENT}

The authors would like to thank Sheng Wang for her help in the course of numerical experiments.

\section{REFERENCES}

[1] E. Bogomol'ny, The stability of classical solutions, Sov. J. Nucl. Phys., Vol. 24, 1976, pp. $449-454$.

[2] H. J. de VEGA and F. SCHAPOSNIK, Electrically charged vortices in nonabelian gauge theories with Chern-Simons term, Phys. Rev. Lett., Vol. 56, 1986, pp. 2564-2566.

[3] J. Fröhlich and P. MarChetTi, Quantum field theory of anyons, Lett. Math. Phys., Vol. 16, 1988, pp. 347-358.

[4] J. FröHLICH and P. MarChETTI, Quantum field theory of vortices and anyons, Commun. Math. Phys., Vol. 121, 1989, pp. 177-223.

[5] D. Gilbarg and N. Trudinger, Elliptic Partial Differential Equations of Second Order, Springer, Berlin, 1977.

[6] J. Hong, Y. KIM and P. PAC, Multivortex solutions of the abelian Chern-Simons-Higgs theory, Phys. Rev. Lett., Vol. 64, 1990, pp. 2230-2233.

[7] R. JACKI, Solitons in Chern-Simons/anyons systems, Preprint.

[8] R. Jackiw, K. Lee and E. Weinberg, Self-dual Chern-Simons solitons, Phys. Rev. D., Vol. 42, 1990, pp. 3488-3499.

[9] R. JACKIW, S.-Y. PI and E. WEINBERG, Topological and non-topological solitons in relativistic and non-relativistic Chern-Simons theory, Preprint.

[10] R. JACKIw and E. Weinberg, Self-dual Cherm-Simons vortices, Phys. Rev. Lett., Vol. 64, 1990, pp. 2234-2237.

[11] A. JAFFe and C. H. TAubes, Vortices and Monopoles, Birkhaüser, Boston, 1980.

[12] B. JuliA and A. ZEE, Poles with both magnetic and electric charges in nonabelian gauge theory, Phys. Rev. D., Vol. 11, 1975, pp. 2227-2232.

[13] H. NiElSEN and P. Olesen, Vortex-line models for dual-strings, Nucl. Phys. B, Vol. 61, 1973, pp. 45-61.

[14] S. Paul and A. Khare, Charged vortices in an abelian Higgs model with Chern-Simons term, Phys. Lett. B, Vol. 174, 1986, pp. 420-422.

[15] S. PAUl and A. KHARE, Charged vortex of finite energy in nonabelian gauge theories with Chern-Simons term, Phys. Lett. B, Vol. 178, 1986, pp. 395-399. 
[16] J. SPRUCK and Y. YANG, The existence of non-topological solitons in the self-dual Chern-Simons theory, Commun. Math. Phys., Vol. 149, 1992, pp. 361-376.

[17] C. TAUBES, On the equivalence of the first and second order equations for gauge theories, Commun. Math. Phys., Vol. 75, 1980, pp. 207-227.

[18] R. WANG, The existence of Chern-Simons vortices, Commun. Math. Phys., Vol. 137, 1991, pp. 587-597.

[19] S. WANG and Y. YANG, Abrikosov's vortices in the critical coupling, SIAM J. Math. Anal., Vol. 23, 1992, pp. 1125-1140.

[20] S. WANG and Y. YANG, Solutions of the generalized Bogomol'nyi equations via monotone iterations, J. Math. Phys., Vol. 33, 1992, pp. 4239-4249.

[21] Y. YANG, Existence, regularity, and asymptotic behavior of the solutions to the GinzburgLandau equations on $\mathbf{R}^{3}$, Commun. Math. Phys., Vol. 123, 1989, pp. 147-161. 